
\title{
25 Research Square \\ The characteristics of echocardiography in ICU patients and its relationship with the outcomes
}

\section{Qian-Yi Peng}

Xiangya Hospital Central South University

\section{Li-Xia Liu}

Hebei Medical University Fourth Affiliated Hospital and Hebei Provincial Tumor Hospital

\section{Qian Zhang}

Guizhou Medical University

\section{Ying Zhu}

Hangzhou First People's Hospital

\section{Hong-Min Zhang}

Peking Union Medical College Hospital

\section{Wan-Hong Yin}

Sichuan University West China Hospital

\section{Wei He}

Beijing Tongren Hospital

\section{Xiu-Ling Shang}

Fujian Provincial Hospital

\section{Yan-Gong Chao}

Tsinghua University

\section{Li-Wen Lv}

People's Hospital of Guangxi Zhuang Autonomous Region

\section{Xiao-Ting Wang}

Peking Union Medical College Hospital

\section{Li-Na Zhang ( $\square$ zln7095@163.com )}

Xiangya Hospital Central South University

\section{Research}

Keywords: Echocardiography, intensive care, acute respiratory failure, shock, post-operation, mortality

Posted Date: August 26th, 2020

DOI: https://doi.org/10.21203/rs.3.rs-64860/v1 
License: (c) (i) This work is licensed under a Creative Commons Attribution 4.0 International License. Read Full License 


\section{Abstract}

Background Echocardiography is widely used for bedside monitoring in ICU. We aimed to describe the characteristics of echocardiography manifestations in different groups of ICU patients, and the association with the outcomes.

Methods This is a prospective multiple-center cohort study took place in 17 ICUs in China. A total of 1682 continuous adult patients admitted between August 31, 2017 to February 16, 2019 who had echocardiography performed within 24 hours in ICU were included. The echocardiography was performed and the echocardiography scores were calculated. Data was analyzed and compared between different outcome and primary indication groups.

Results The overall 28 -day mortality was $15.76 \%$. Left ventricle enlargement and other echocardiogram abnormalities were more common in the non-survivors than the survivors. The EF value was lower, and the decrease in EF value was more frequent in the non-survivors than the survivors. The echocardiography score was significantly higher in the non-survivors than the survivors. The incidence of right ventricle enlargement and EF decrease was high in ICU patients (26.52\% and $26.93 \%$ respectively). The independent risk factors for 28-day mortality was APACHE II, the length of MV, the length of ICU stay, oxygenation index, right ventricle wall thickening, IVC diameter, IVC variability, and the echocardiography score.

Conclusions The incidence of cardiac dysfunction is high in ICU patients. The echocardiography score was significantly higher in the non-survivors than the survivors, and was an independent risk factor for 28-day mortality. Echocardiography is a convenient bedside monitoring method which deserves widely use in ICUs.

Trial registration: Chinese Clinical Trial Registry (ChiCTR-DDD-17012391). Registered 2017.08.17. http://www.chictr.org.cn/showprojen.aspx?proj=21127.

\section{Background}

Acute and chronic cardiac dysfunction is common in critically ill patients. Early detection of these signs is important for clinical management. Echocardiography is a very useful tool in the evaluation, treatment, and follow-up of intensive care unit (ICU) patients. Echocardiography is convenient for bedside monitoring to determine the type and state of shock, cardiac function, volume status, etc., therefore, it is increasingly widely used in ICUs.

In 2010, a statement on the subject of point-of-care cardiac evaluations being performed by noncardiologists was issued ${ }^{[1]}$, which termed the focused cardiac ultrasound (FOCUS). Later on, in 2013, the American Society of Echo decided that FOCUS should be used as an adjunct to the physical exam in an emergency setting ${ }^{[1]}$. Many different types of FOCUS exams for rapid evaluation of emergency or ICU patients have been introduced ${ }^{[2,3]}$, including the rapid ultrasound in shock (RUSH) protocol ${ }^{[4]}$, the focus- 
assessed transthoracic echocardiography (FATE) and the advanced FATE protocol ${ }^{[5]}$, the fluid administration limited by lung sonography (FALLS) protocol ${ }^{[6]}$, the focused assessment with sonography for trauma (FAST) and extended FAST (eFAST) ${ }^{[7]}$, lung ultrasound study (CCROSS) protocol ${ }^{[8]}$, and the critical care chest ultrasonic examination (CCUE) protocol ${ }^{[9]}$, etc..

Most of these protocols use similar rapid echocardiography examinations including five standard views. Previous studies which applied these protocols reported early detection of etiology and complications, the improvement of the bedside treatment of critically ill patients, and reduce of the length of ICU stay ${ }^{[10-12]}$. However, most of these studies were singer center studies with relatively small sample size. The objectives of our study were to describe the characteristics of echocardiography manifestation in different groups of ICU patients, created a easy echocardiography score, and analyze their associations with outcomes.

\section{Methods}

\section{Ethics statement}

This prospective multiple-center cohort study was registered on the Chinese Clinical Trial Registry (ChiCTR-DDD-17012391), and was permitted by the Ethics Committee of each participating hospital. Informed consent was obtained from all participants or their family members.

\section{Study design and patient selection}

Data collection took place in 24 ICUs of tertiary public hospitals in China. Continuous adult patients admitted between August 31, 2017 to February 16, 2019 who had LUS performed within 24 hours in ICU were included. A total of 1913 patients were accrued during this time, twenty-four patients were exclude for age less than 18-years old and 187 patients were excluded for incomplete data records. Finally, the data of 1682 patients were used for analysis.

\section{Data collection}

Data that was prospectively collected following inclusion. Briefly, these data included demographics, heart rate (HR), mean arterial pressure (MAP), lactate (Lac), Acute Physiology and Chronic Health Evaluation (APACHE) II scores, and measures of oxygenation indexes $\left(\mathrm{PaO}_{2} / \mathrm{FiO}_{2}\right.$ ratio) within 24 hours of ICU admission, length of mechanical ventilation (MV), length of ICU stay and 28-day mortality.

\section{Echocardiogram measurements}

Echocardiogram measurements were performed using the portable ultrasound machine (GE, Mindray or Sonosite) with a phased-array probe $(2.5 \mathrm{MHz})$. All patients were placed in the supine position for echocardiogram measurements. Five views were examined: parasternal view long axis, parasternal view short axis, apical view, subxiphoid view, and inferior vena cava (IVC) long axis. Left atrium enlargement is 
defined as $>33 \mathrm{~mm}$. Right atrium enlargement is defined as: $>45 \times 45 \mathrm{~mm}$ from the apical view. Left ventricle (LV) enlargement is defined as $>55 \mathrm{~mm}$ for male and $>50 \mathrm{~mm}$ for female. Right ventricle (RV) enlargement is defined as the ratio of RV/LV (the end-diastolic area) $>0.6$. Left ventricular wall thickening is defined as $>12 \mathrm{~mm}$. Right ventricular wall thickening is defined as $>7 \mathrm{~mm}$. Ejection fraction (EF) is measured in the left ventricular long-axis view or papillary muscle short-axis view, with the cursor perpendicular to the interventricular septum. IVC diameter and variability were measured from subxiphoid, two centimeters distal from the right atrium ${ }^{[13]}$. IVC variability was defined as IVCmax-IVCmin)/IVCmax for spontaneous respiratory patients, and IVCmax-IVCmin)/IVCmin for MV patients. For spontaneous respiratory patients, IVC variability was classified as large when $>50 \%$, medium when $10-50 \%$, and mall when $<10 \%[9]$. For spontaneous respiratory patients, IVC variability was classified as large when $>18 \%$, medium when $10-18 \%$, and mall when $<10 \%{ }^{[14]}$. Other echocardiogram abnormalities including pericardial effusion, heart valve abnormalities, pulmonary hypertension, ventricular wall segmental dyskinesia, etc. The above mentioned abnormal findings in echocardiography were scored: (1) Left/Right atrium enlargement: score 1; (2) Left ventricle enlargement: score 1; (3) Right ventricle enlargement: $R V / L V<0.6$, score $0 ; R V / L V=0.6-1.0$, score 1; $R V / L V>1.0$, score 2; (4)Left/Right ventricular wall thickening: score 1 ; (5) IVC diameter: $1.0-1.9$, score 0 ; $<1.0$ or $\geq 2.0$, score 1 ; (6) IVC variability: Medium: score 0 ; large or small: score 1 ; (7) EF: $>55 \%$, score $0 ; 30-55 \%$, score $1 ;<30 \%$, score 2 ; (8) Other echocardiogram abnormalities: score 1 . The echocardiography score was calculated by the sum of these scores. All the measurements were performed by two constant intensivists who have been trained by Chinese Critical Ultrasound Study Group (CCUSG) in each participating ICUs. Echocardiography was first measured by one of them, and then confirmed by another person (blinded to the first). When their results were similar, the data was recorded; otherwise, a third person would perform the measurements again, and the two most similar results was record.

\section{Statistics}

Continuous variables were reported as mean \pm standard deviation (SD), and were compared using the Student's $t$ test or one-way analysis of variance (ANOVA) if a normal distribution or a homogeneity of variance was detected. Otherwise, the Mann-Whitney U test or Kruskal-Wallis test was used. Categorical variables were compared using the $\chi^{2}$ test. A 2-tailed $\mathrm{P}<0.05$ was considered statistically significant.

Risk factors for 28-day mortality were assessed by multiple logistic regression based on the enter method. Results of the multivariate logistic regression analysis were summarized by estimating odds ratios (ORs) and respective $95 \%$ confidence intervals (Cls). The predictive power of ultrasound score for 28-day mortality was assessed by the area under the receiver operator characteristic (AuROC) curve. All statistical analysis was performed using SPSS version 19 for Windows (SPSS Inc., USA).

\section{Results}

A total of 1682 patients were included in this study. The mean age of the participants was $59.48 \pm 17.363$ years, with male composing $58.32 \%$ of the cohort $(n=981)$. The primary indications for ICU admission 
included: respiratory failure $(n=533,31.69 \%)$, shock $(n=288,17.12 \%)$, post-operation $(n=759,45.12 \%)$, and others $(n=265,15.76 \%)$. The mean APACHE II score was $15.32 \pm 8.452$. The mean length of MV was $91.12 \pm 213.319$ hours, and the mean length of ICU stay was $13.58 \pm 60.156$ days. The overall 28-day mortality was $15.76 \%(265 / 1682)$. (Table 1 )

\section{Differences between survivors and non-survivors}

Among the overall patients, the ages of survivors were significantly younger than non-survivors $(P=0.013)$. APACHE II scores and the length of MV were significantly lower in the survivors than the nonsurvivors ( $p<0.001$ and $p<0.001$ respectively). The HR at admission was higher and the MAP was lower in the non-survivors than the survivors ( $p<0.001$ and $p<0.001$ respectively). The Lac was higher and the oxygenation index was lower in the non-survivors than the survivors ( $<<0.001$ and $p<0.001$ respectively). In echocardiography examination, left ventricle enlargement and other echocardiogram abnormalities were more frequent in the non-survivors than the survivors ( $p=0.041$ and $p<0.001$ respectively). The EF value was significantly lower, and decrease in $\mathrm{EF}$ value is more frequent in the non-survivors than the survivors ( $p<0.001$ and $p<0.001$ respectively). The echocardiography score was significantly higher in the non-survivors than the survivors $(p=0.022)$. (Table 2 )

For subgroup analysis, in patients with acute respiratory failure $(n=533)$, APACHE II scores were significantly lower in the survivors than the non-survivors $(p<0.001)$, while the age, length of $M V$ and length of ICU stay were similar between groups. The Lac was higher in the non-survivors than the survivors ( $p<0.001)$. In echocardiography examination, IVC diameter $<1.0 \mathrm{~cm}$ was more frequent in the non-survivors than the survivors $(p=0.002)$. IVC variability large or small was more frequent in the nonsurvivors than the survivors ( $p<0.001$ and $p=0.034$ respectively). There was no significant difference on the echocardiography score between the non-survivors and the survivors $(p=0.454)$. (Table 3$)$

In patients with shock $(\mathrm{n}=288)$, APACHE II scores were significantly lower in the survivors than the nonsurvivors $(p<0.001)$, while the age, length of MV and length of ICU stay was similar between groups. The Lac was higher in the non-survivors than the survivors $(p<0.001)$. In echocardiography examination, other echocardiogram abnormalities were more frequent in the non-survivors than the survivors $(p=0.044)$. There was no significant difference on the echocardiography score between the non-survivors and the survivors $(p=0.685)$. (Table 4$)$

In postoperative patients ( $n=759)$, ages, APACHE II scores, and the length of MV were significantly lower in the survivors than the non-survivors ( $p=0.010, P<0.001$ and $p<0.001$ respectively). The HR and the Lac was higher in the non-survivors than the survivors ( $p=0.035$ and $p=0.023$ respectively). In echocardiography examination, left ventricle enlargement, left ventricular wall thickening, right ventricular wall thickening and other echocardiogram abnormalities were more frequent in the non-survivors than the survivors $(p<0.001, p=0.042, p=0.003$ and $p=0.006$ respectively). The $E F$ value was significantly lower, and $\mathrm{EF}<30 \%$ is more frequent in the non-survivors than the survivors ( $\mathrm{p}=0.014$ and $p<0.001$ respectively). The echocardiography score was significantly higher in the non-survivors than the survivors $(p=0.001)$. (Table 5) 


\section{The risk factors of 28-day mortality}

Using multivariate analysis, the independent risk factors for 28-day mortality was APACHE II (OR=0.903, $\mathrm{p}<0.001,95 \% \mathrm{Cl} 0.873-0.933)$, the length of $\mathrm{MV}(\mathrm{OR}=0.994, \mathrm{p}<0.001,95 \% \mathrm{Cl} 0.991-0.997)$, the length of ICU stay (OR=1.159, $p<0.001,95 \% \mathrm{Cl} 1.083-1.242)$, oxygenation index $(\mathrm{OR}=1.003, p=0.013,95 \% \mathrm{Cl} 1.001-$ 1.005), IVC diameter ( $O R=1.756, p=0.024,95 \% \mathrm{Cl} 1.075-2.868)$, IVC variability $(\mathrm{OR}=1.797, \mathrm{p}=0.017,95 \% \mathrm{Cl}$ 1.112-2.904), and the echocardiography score ( $\mathrm{OR}=0.443, \mathrm{p}=0.016,95 \% \mathrm{Cl} 0.228-0.861)$. To predict the 28-day mortality of ICU patients, the AuROC of the echocardiography score were 0.595 . (Table 6)

\section{Discussion}

Critical care ultrasound has gained its place as an effective and convenient monitoring tool, which become the "visual stethoscope" of the 21 st century ${ }^{[15,16]}$. Echocardiography is an important part of critical care ultrasound, which allows for an anatomical, functional, and hemodynamic assessment of the heart, and has revolutionized the bedside assessment of ICU patients. Many different types of FOCUS exams have been introduced to the emergency departments or ICUs. Previous studies have reported that FOCUS performed by emergency medicine residents is comparable to echocardiography performed by cardiologists. Therefore, echocardiography is a reliable tool and screening test for cardiac abnormalities ${ }^{[17]}$. After a brief standard training in using echocardiographic system, intensivists can successfully performed and correctly interpreted a focused TTE for critically ill patients ${ }^{[18]}$. Chest ultrasonography is a valid bedside diagnostic aid to the management of acute respiratory diseases in older patients ${ }^{[19]}$. FOCUS can be an important tool in the initial evaluation of emargency patients with suspected pulmonary embolism and abnormal vital signs ${ }^{[20]}$. In cardiac-arrest patients, after return of spontaneously circulation, FOCUS could be included in post-resuscitation care as an adjunctive diagnostic measure ${ }^{[21]}$. CCUE-plus protocol was effective in the etiological diagnosis in patients with dyspnea and/or hemodynamic instability caused by abdominal abnormalities ${ }^{[22]}$. However, the prognostic value of echocardiography in ICU patients is seldom studied. It was reported that CCUE protocol could improve the bedside treatment of critically ill patients, and reduce ICU stay ${ }^{[10]}$. A FOCUS examination for right ventricular strain performed by emergency care practitioners was a significant predictor of in-hospital adverse outcomes among patients diagnosed with pulmonary embolism in the Emergency Department ${ }^{[23]}$. But the role of echocardiography performed early during admission in general ICU patients has not been assessed in a multiple center cohort. In this study, we described the characteristics of echocardiography manifestation in different groups of ICU patients, and analyzed their associations with outcomes.

This study included 1682 patients from the ICUs in different parts of China. The mean age of the participants was $59.48 \pm 17.363$ years, and the mean APACHE II score was $15.32 \pm 8.452$. The overall 28day mortality was $15.76 \%$ (265/1682), which is in line with the general level of ICU patients. We applied echocardiography to these general ICU patients with different primary indications, and analyzed its relationship with the 28-day mortality. Results showed APACHE II scores, the length of MV, HR, MAP and 
Lac were significantly different between the non-survivors and the survivors. Left ventricle enlargement and other echocardiogram abnormalities were more frequent in the non-survivors than the survivors. The incidence of right ventricle enlargement (446/1682, 26.52\%) and EF decrease (453/1682, 26.93\%) was high in ICU patients. The EF value was significantly lower, and the decrease in EF value was more frequent in the non-survivors than the survivors. These results indicated that the incidence of cardiac dysfunction was high in ICU patients. Echocardiography at the admission of ICU helps early detection of cardiac abnormalities.

Then we analyzed the characteristic of echocardiography in subgroups with different primary diseases. In patients with acute respiratory failure, the incidence of right ventricle enlargement was even higher $(178 / 533,33.40 \%)$. IVC variability abnormal was more frequent in the non-survivors than the survivors. IVC diameter is a useful mirror of the right atrial pressure (RAP), and IVC variability is a reliable index for assessing fluid responsiveness ${ }^{[13,14]}$. This indicated that unsuitable volume status might be an important factor for the mortality of acute respiratory failure patients. However, the echocardiography score was not significantly different between the non-survivors and the survivors. A probable reason was that cardiac abnormalities were common in patients with acute respiratory failure, thus the difference was not obvious between groups.

In patients with shock, in echocardiography examinations, only other echocardiogram abnormalities were significantly different between the non-survivors than the survivors. Other cardiac abnormalities including pericardial effusion, heart valve abnormalities, pulmonary hypertension, ventricular wall segmental dyskinesia, were important clinical conditions leading to heart failure and deterioration of diseases. In shock patients, there was no significant difference on EF values between groups, probably because most of the shock patients included in this study were septic shock instead of cardiogenic shock. Also, the echocardiography score was not significantly different between the nonsurvivors and the survivors, probably because cardiac abnormalities were common in patients with shock.

In postoperative patients, left ventricle enlargement, left ventricular wall thickening, right ventricular wall thickening and other echocardiogram abnormalities were more frequent in the non-survivors than the survivors, indicating that primary heart diseases were important factors for the mortality of postoperative patients. The $\mathrm{EF}$ value was significantly lower, and $\mathrm{EF}<30 \%$ is more frequent in the non-survivors than the survivors, indicating that cardiac systolic dysfunction was also important for the outcome of patients. Previous studies has reported that LV ejection fraction $<45 \%$ was associated with the absence of early recovery and less favorable 1-year outcome in patients with Takotsubo Syndrome ${ }^{[24]}$. A scoring system including EF is a predictor of 30-day mortality risks for ST-Elevation Myocardial Infarction patients ${ }^{[25]}$. The echocardiography score was significantly higher in the non-survivors than the survivors. These results indicated that echocardiography screen was important for post-operative patients. And abnormal findings in echocardiography may help divide the non-survivors and survivors. 
When analyzing the risk factors for 28-day mortality, the independent risk factors was APACHE II, the length of MV, the length of ICU stay, oxygenation index, right ventricular wall thickening, IVC diameter, IVC variability and the echocardiography score. These data indicated that volume status and fluid responsiveness were critical for the mortality of ICU patients. However, a previous study using IVC variability $<20 \%$ as the end-point of fluid resuscitation in septic shock requiring ventilatory support did not found significant difference in the amount of fluid infused, time to reach end-point and mortality with the regular care group. As there are few studies on the relationship between IVC variability and mortality, it deserves further study.

In this study, we developed a scoring system by assessing atrium and ventricle enlargement, ventricular wall thickening, IVC diameter and variability, EF, and other echocardiogram abnormalities, therefore to generally evaluate the structural, systolic, diastolic and volumic abnormalities. We found that the echocardiography score was significantly higher in the non-survivors than the survivors. Also, it was an independent risk factor for 28-day mortality. These results indicated that echocardiography makers that reveal the general cardiac function could help to predict the outcome. However, the AuROC of echocardiography score for predicting the 28-day mortality was only 0.595 , indicating that it is not a reliable outcome predicting marker. Therefore, this echocardiography score may be better used in combination with other risk factors of mortality.

This study is a multiple center study that comprehensively investigated the characteristics of echocardiography manifestations and their prognostic importance in a larger cohort of ICU patients. However, our study has some limitations. First, as many clinical indicators were not completely recorded in different participating sites, we only analyzed the most complete baseline data including the age, gender, primary indications, HR, MAP, Lac, APACHE II scores, length of MV, length of ICU stay, and 28-day mortality. However, many laboratory indexes were not analyzed because of too many missing data. Second, most participating ICUs are general ICUs, with relatively more surgical patients and fewer internal medical patients. Therefore, a large proportion of the included patients were postoperative patients with less severe conditions.

\section{Conclusions}

This large multiple center study demonstrated that the incidence of cardiac dysfunction is high in ICU patients. the IVC variability is an independent risk factors for 28-day mortality. Echocardiography is a convenient bedside monitoring method which deserves widely use in ICUs.

\section{References}

1. Glaser J, Conti B, Murthi S. Ultrasonography in the ICU. Springer; 2015. Cardiac ultrasound in the intensive care unit: point-of-care transthoracic and transesophageal echocardiography.

2. 10.7759 /cureus. 4612 
Patel AR, Patel AR, Singh S, Singh S, Khawaja I. Cardiac Ultrasound in the Intensive Care Unit: A Review. Cureus. 2019 May 7;11(5):e4612. doi: 10.7759/cureus.4612.

3. Longobardo L, Zito C, Carerj S, Caracciolo G, Bijoy K. Khandheria. Role of Echocardiography in the Intensive Care Unit: Overview of the Most Common ClinicalScenarios. J Patient Cent Res Rev. 2018 Summer;5(3):239-43. doi:10.17294/2330-0698.1631. Published online 2018 Jul 30.

4. Garduño-López J, García-Cruz E, Baranda-Tovar FM. Cardiac, cerebral, renal, optic nerve, and lung ultrasound study (CCROSS) protocol. Arch Cardiol Mex. 2019;89(1):126-37. doi:10.24875/ACME.M19000035.

5. Nagre AS. Focus-assessed transthoracic echocardiography: Implications in perioperative and intensive care. Ann Card Anaesth. 2019 Jul-Sep;22(3):302-8. doi:10.4103/aca.ACA_88_18.

6. Perera P, Mailhot T, Riley D, Mandavia D. The RUSH exam: Rapid Ultrasound in Shock in the evaluation of the critically III. Emerg Med Clin N Am. 2010;28:29-56.

7. Lichtenstein D, Karakitsos D. Integrating lung ultrasound in the hemodynamic evaluation of acute circulatory failure (the fluid administration limited by lung sonography protocol). J Crit Care. 2012;27:533.

8. Giraldo-Restrepo JA, Serna-Jiménez TJ. The FAST and extended FAST examinations. Col J Anesthesiol. 2015;43(4):299-306.

9. Zhang LN, Zhang HM, Cao YG, Yin WH, He W, Zhu R, Ding X, Liu LX, Wu J, Li L, Liu HT, Ai YH, Wang XT, Chinese Critical Ultrasound Study Group (CCUSG). Ten Basic Principles about Critical Ultrasonography: Critical Care Practitioners Need to Know. Chin Med J (Engl). 2017 Jul;5(13):16104. doi:10.4103/0366-6999.208229. 130 ).

10. Wang X, Liu D, He H, Du W, Zhang H, Liu Y, et al. Using critical care chest ultrasonic examination in emergency consultation: A pilot study. Ultrasound Med Biol. 2015;41:401-6.

doi:10.1016/j.ultrasmedbio.2014.09.010.

11. Netherton S, Milenkovic V, Taylor M, Davis PJ. Diagnostic accuracy of eFAST in the trauma patient: a systematic review and meta-analysis. CJEM. 2019 Nov;21(6):727-38. doi:10.1017/cem.2019.381.

12. Lichtenstein DA. BLUE-protocol and FALLS-protocol: two applications of lung ultrasound in the critically ill. Chest. 2015 Jun;147(6):1659-70. doi:10.1378/chest.14-1313.

13. Lanspa MJ, Grissom CK, Hirshberg EL, Jones JP, Brown SM. Applying dynamic parameters to predict hemodynamic response to volume expansion in spontaneously breathing patients with septic shock. Shock. 2013 Feb;39(2):155-60. doi:10.1097/SHK.0b013e31827f1c6a.

14. Barbier C, Loubières $Y$, Schmit C, Hayon J, Ricôme JL, Jardin F, Vieillard-Baron A. Respiratory changes in inferior vena cava diameter are helpful in predicting fluid responsiveness in ventilated septic patients. Intensive Care Med. 2004 Sep;30(9):1740-6.

15. Peng QY, Zhang LN, Ai ML, Li L, Hu CH, Zhang YX, Liu W, Feng Q, Zou Y, Ai YH, Chinese Critical Ultrasound Study Group. Common Carotid Artery Sonography Versus Transthoracic Echocardiography for Cardiac Output Measurements in Intensive Care Unit Patients. J Ultrasound Med. 2017 Sep;36(9):1793-9. doi:10.1002/jum.14214. 
16. 10.11817/j.issn.1672-7347.2018.04.018

Cao L, Zhang L, Ai M, Li L, Tian D, Sun Y, Deng L. Application of radial arterial puncture cannulation under ultrasonic guidance in patients with critical diseases. Zhong Nan Da Xue Xue Bao Yi Xue Ban. 2018 Apr 28;43(4):447-451. doi: 10.11817/j.issn.1672-7347.2018.04.018.

17. $10.1007 / s 40477-017-0246-5$

Farsi D, Hajsadeghi S, Hajighanbari MJ, Mofidi M, Hafezimoghadam P, Rezai M, Mahshidfar B, Abiri S, Abbasi S. Focused cardiac ultrasound (FOCUS) by emergency medicine residents in patients with suspected cardiovascular diseases. J Ultrasound. 2017 May 2;20(2):133-138. doi: 10.1007/s40477017-0246-5. eCollection 2017 Jun.

18. Zhang LN, Ai YH, Liu ZY, Tian CH, Zhu JX. Feasibility of focused transthoracic echocardiography in intensive care unit performed by intensivists. Zhongguo Wei Zhong Bing Ji Jiu Yi Xue. 2012 Dec;24(12):739-41.

19. 10.1016/j.jamda.2019.06.018

Ticinesi A, Scarlata S, Nouvenne A, Lauretani F, Incalzi RA, Ungar A; GRETA (Gruppo di Ricerca sull'Ecografia Toracica nell'Anziano) Group of the Italian Society of Gerontology and Geriatrics (SIGG). The Geriatric Patient: The Ideal One for Chest Ultrasonography? A Review From the ChestUltrasound in the Elderly Study Group (GRETA) of the Italian Society of Gerontology and Geriatrics (SIGG). J Am Med Dir Assoc. 2019 Aug 6. pii: S1525-8610(19)30513-4. doi:

10.1016/j.jamda.2019.06.018.

20. Daley JI, Dwyer KH, Grunwald Z, Shaw DL, Stone MB, Schick A, Vrablik M, Kennedy Hall M, Hall J, Liteplo AS, Haney RM, Hun N, Liu R, Moore CL. Increased Sensitivity of Focused Cardiac Ultrasound for Pulmonary Embolism in Emergency Department Patients With Abnormal Vital Signs. Acad Emerg Med. 2019 Nov;26(11):1211-20. doi:10.1111/acem.13774.

21. Elfwén L, Hildebrand K, Schierbeck S, Sundqvist M, Ringh M, Claesson A, Olsson J, Nordberg P. Focused cardiac ultrasound after return of spontaneous circulation in cardiac-arrest patients. Resuscitation. 2019 Sep;142:16-22. doi: 10.1016/j.resuscitation.2019.06.282.

22. China Critical Ultrasound Study Group (CCUSG)

10.3760/cma.j.issn.0578-1426.2017.08.006

Li L, Ai YH, Jiang S, Zhang YX, Hu CH, Ai ML, Ma XH, Liu ZY, Zhang LN. China Critical Ultrasound Study Group (CCUSG). The application of Critical Care Chest Ultrasonic Evaluation-plus Protocol in the etiological diagnosis of dyspnea and/or hemodynamic instability caused by abdominal abnormality. Zhonghua Nei Ke Za Zhi. 2017 Aug 1;56(8):583-587. doi: 10.3760/cma.j.issn.05781426.2017.08.006.

23. Taylor RA, Davis J, Liu R, Gupta V, Dziura J, Moore CL. Point-of-care focused cardiac ultrasound for prediction of pulmonary embolism adverse outcomes. J Emerg Med. 2013 Sep;45(3):392-9. doi:10.1016/j.jemermed.2013.04.014.

24. Jurisic S, Gili S, Cammann VL, Kato K, Szawan KA, D'Ascenzo F, et al. Clinical Predictors and Prognostic Impact of Recovery of Wall Motion Abnormalities in Takotsubo Syndrome: Results From 
the International Takotsubo Registry. J Am Heart Assoc. 2019 Nov;5(21):e011194.

doi:10.1161/JAHA.118.011194. 8 ) .

25. Ginanjar E, Yamin M, Wijaya IP, Harimurti K. Predictors of 30-day Mortality in ST-Elevation Myocardial Infarction (STEMI) Patients. Acta Med Indones. 2019 Jul;51(3):238-44.

26. Mekontso Dessap A, Boissier F, Charron C, Bégot E, Repessé X, Legras A, Brun-Buisson C, Vignon P, Vieillard-Baron A. Acute cor pulmonale during protective ventilation for acute respiratory distress syndrome: prevalence, predictors, and clinical impact. Intensive Care Med. 2016 May;42(5):862-70. doi:10.1007/s00134-015-4141-2.

\section{List Of Abbreviations}

Focused cardiac ultrasound (FOCUS); rapid ultrasound in shock (RUSH); focus-assessed transthoracic echocardiography (FATE); fluid administration limited by lung sonography (FALLS); focused assessment with sonography for trauma (FAST); critical care chest ultrasonic examination (CCUE); heart rate (HR); mean arterial pressure (MAP); lactate (Lac); Acute Physiology and Chronic Health Evaluation (APACHE); mechanical ventilation (MV); inferior vena cava (IVC); left ventricle (LV); right ventricle (RV); ejection fraction (EF); standard deviation (SD); one-way analysis of variance (ANOVA); odds ratios (ORs); confidence intervals (Cls); area under the receiver operator characteristic (AuROC).

\section{Declarations}

- -Ethics approval and consent to participate: This prospective multiple-center cohort study was registered on the Chinese Clinical Trial Registry (ChiCTR-DDD-17012391), and was permitted by the Ethics Committee of each participating hospital.

- -Consent for publication: This paper has not been published elsewhere in whole or in part. All authors have read and approved the content, and agree to submit it for consideration for publication in your journal.

- -Availability of data and materials: Available.

- -Competing interests: There was no conflict of interest to be declared.

- -Funding: Li-Na Zhang receives a funding from National Natural Science Foundation of China (Grant no. 81873956). Qian-Yi Peng receives a funding from National Natural Science Foundation of China (Grant no. 81974285).

- -Authors' contributions:

Qian-Yi Peng drafted this paper, performed lung ultrasound, collected and analyzed the data. Li-Xia Liu, Qian Zhang, Ying Zhu, Hong-Min Zhang, Wan-Hong Yin, Wei He, Xiu-Ling Shang, Yan-Gong Chao, Li-Wen Lv, and Xiao-Ting Wang performed lung ultrasound and collected data in their ICUs. Li-Na Zhang designed and supervised this study, and modified the paper. 
We would like to thank the following persons for their assistance in this work: $\mathrm{Li} \mathrm{Li}$, Cheng-Huan Hu, MeiLin Ai (Department of Critical Care Medicine, Xiangya Hospital, Central South University), Tong-Juan Zou, Yi Li (Department of Critical Care Medicine, West China Hospital of Sichuan University), Hua Zhao, Huan Chen (Department of Critical Care Medicine, Peking Union Medical College Hospital), Yang Liu, Na Liu (Department of Critical Care Medicine, Beijing Tongren Hospital), Qin-Bing Zeng (Department of Critical Care Medicine, the First Affiliated Hospital of Tsinghua University), Mu-Shan Ai (Department of Critical Care Medicine, Daping Hospital Affiliated to the Third Military Medical University), Min-Jia Wang (Department of Critical Care Medicine, Zhejiang Hospital), Ran Zhu (Department of Critical Care Medicine, the First Affiliated Hospital of China Medical University), and Jia-Wei Ren (the Fourth Affiliated Hospital of Hebei Medical University).

\section{Tables}

Table 1 Baseline characteristics and outcomes of patients included. 


\begin{tabular}{|lll|}
\hline Variable & \multicolumn{2}{l|}{ Number (percentage) } \\
\hline Age $(\mathrm{y})$ & $59.48 \pm 17.363$ & (Mean \pm SD) \\
\hline Age groups & & \\
\hline$<50$ & 468 & $27.82 \%$ \\
\hline $50-59$ & 289 & $17.18 \%$ \\
\hline $60-69$ & 391 & $23.25 \%$ \\
\hline$\geq 70$ & 534 & $31.75 \%$ \\
\hline Gender & & \\
\hline Male & 981 & $58.32 \%$ \\
\hline Female & 701 & $41.68 \%$ \\
\hline Primary indication & & $31.69 \%$ \\
\hline Respiratory failure & 533 & $17.12 \%$ \\
\hline Shock & 288 & $45.12 \%$ \\
\hline Postoperation & 759 & $15.76 \%$ \\
\hline Others & 265 & \\
\hline APACHEII & $15.32 \pm 8.452$ & \\
\hline Length of MV (h) & $91.12 \pm 213.319$ \\
\hline Length of ICU stay (d) & $13.58 \pm 60.156$ & \\
\hline 28-day mortality & $265 / 1682$ & $15.76 \%$ \\
\hline
\end{tabular}

Table 2 Clinical characteristics, outcomes and echocardiography manifestations stratified by survival status 28 days after ICU admission in ICU patients. 


\begin{tabular}{|c|c|c|c|}
\hline Variable & Non-survivors & Survivors & $P$ \\
\hline Age (y) & $61.89 \pm 17.022$ & $59.03 \pm 17.395$ & $0.014^{\star}$ \\
\hline $\mathrm{HR}$ & $101.99 \pm 26.193$ & $91.74 \pm 24.195$ & $<0.001^{*}$ \\
\hline MAP & $87.131 \pm 17.9910$ & $91.174 \pm 16.6552$ & $<0.001^{*}$ \\
\hline Lac & $3.529 \pm 3.8676$ & $2.187 \pm 2.4502$ & $<0.001^{*}$ \\
\hline Oxygenation Index & $201.657 \pm 146.1023$ & $263.172 \pm 115.2306$ & $<0.001^{*}$ \\
\hline APACHEII & $23.13 \pm 8.687$ & $13.86 \pm 7.562$ & $<0.001^{*}$ \\
\hline Lenth of MV (h) & $147.74 \pm 143.597$ & $80.53 \pm 222.406$ & $<0.001 *$ \\
\hline Lenth of ICU stay (d) & $16.15 \pm 70.217$ & $13.10 \pm 58.097$ & 0.450 \\
\hline Left Atrium Enlargement & $31 / 265(11.70 \%)$ & 165/1417 (11.64\%) & 0.980 \\
\hline Right Atrium Enlargement & $32 / 265(12.08 \%)$ & 139/1417 (9.81\%) & 0.263 \\
\hline Left Ventricle Enlargement & $26 / 265(9.81 \%)$ & $90 / 1417(6.35 \%)$ & $0.041^{*}$ \\
\hline Right Ventricle Enlargement & & & 0.075 \\
\hline $\mathrm{RV} / \mathrm{LV}<0.6$ & $180 / 265(67.92 \%)$ & $1062 / 1417$ (74.95\%) & \\
\hline$R V / L V=0.6-1.0$ & $76 / 265(28.68 \%)$ & $324 / 1417(22.87 \%)$ & \\
\hline $\mathrm{RV} / \mathrm{LV}>1.0$ & $9 / 265(3.40 \%)$ & $37 / 1417(2.61 \%)$ & \\
\hline Left Ventricular Wall Thickening & $50 / 265(18.87 \%)$ & 202/1417 (14.26\%) & 0.053 \\
\hline Right Ventricular Wall Thickening & $22 / 265(8.30 \%)$ & 75/1417 (5.29\%) & 0.054 \\
\hline IVC diameter (cm) & & & 0.065 \\
\hline$<1.0$ & $60 / 265(22.64 \%)$ & 261/1417 (18.42\%) & \\
\hline $1.0-1.9$ & $135 / 265$ (50.94\%) & $830 / 1417(58.57 \%)$ & \\
\hline$\geq 2.0$ & $70 / 265$ (26.42\%) & $326 / 1417$ (23.01\%) & \\
\hline IVC variability & & & 0.076 \\
\hline Large & $77 / 265$ (29.06\%) & $322 / 1417(22.72 \%)$ & \\
\hline Medium & $105 / 265$ (39.62\%) & $631 / 1417(44.53 \%)$ & \\
\hline Small & $83 / 265$ (31.32\%) & $464 / 1417$ (32.75\%) & \\
\hline EF & & & $<0.001^{*}$ \\
\hline$>55 \%$ & $160 / 265$ (60.38\%) & 1069/1417 (75.44\%) & $<0.001^{*}$ \\
\hline $30-55 \%$ & $86 / 265$ (32.45\%) & $324 / 1417$ (22.87\%) & 0.001 \\
\hline
\end{tabular}




\begin{tabular}{|llll|}
$<30 \%$ & $19 / 265(7.17 \%)$ & $32 / 1417(2.26 \%)$ & $<0.001^{*}$ \\
\hline EF value & $53.288 \pm 15.7779$ & $59.119 \pm 13.2880$ & $<0.001^{*}$ \\
\hline Other Echocardiogram Abnomalities & $45 / 265(16.98 \%)$ & $145 / 1417(10.23 \%)$ & $0.001^{*}$ \\
\hline Echocardiography Score & $2.69 \pm 1.905$ & $2.10 \pm 1.809$ & $0.022^{*}$ \\
\hline
\end{tabular}

Table 3 Clinical characteristics, outcomes and echocardiography manifestations stratified by survival status $\mathbf{2 8}$ days after ICU admission in patients with acute respiratory failure $(\mathrm{N}=533)$ 


\begin{tabular}{|c|c|c|c|}
\hline Variable & Non-survivors & Survivors & $\mathrm{P}$ \\
\hline Age (y) & $61.94 \pm 17.658$ & $61.29 \pm 17.473$ & 0.700 \\
\hline$H R$ & $103.45 \pm 26.721$ & $100.91 \pm 25.000$ & 0.303 \\
\hline MAP & $87.131 \pm 17.9910$ & $91.174 \pm 16.6552$ & 0.055 \\
\hline Lac & $3.173 \pm 3.2847$ & $2.171 \pm 2.2137$ & $0.001 *$ \\
\hline Oxygenation Index & $173.700 \pm 143.2742$ & $200.006 \pm 98.7661$ & 0.090 \\
\hline APACHEII & $23.69 \pm 8.415$ & $17.89 \pm 7.231$ & $<0.001^{\star}$ \\
\hline Lenth of MV (h) & $162.52 \pm 138.278$ & $181.40 \pm 370.008$ & 0.392 \\
\hline Lenth of ICU stay (d) & $13.66 \pm 46.614$ & $25.27 \pm 96.707$ & 0.063 \\
\hline Left Atrium Enlargement & $16 / 150(10.67 \%)$ & $60 / 383(15.67 \%)$ & 0.138 \\
\hline Right Atrium Enlargement & $16 / 150(10.67 \%)$ & $46 / 383(12.01 \%)$ & 0.663 \\
\hline Left Ventricle Enlargement & $15 / 150(10.00 \%)$ & $32 / 383(8.36 \%)$ & 0.547 \\
\hline Right Ventricle Enlargement & & & 0.906 \\
\hline $\mathrm{RV} / \mathrm{LV}<0.6$ & $102 / 150(68.00 \%)$ & $253 / 383(66.06 \%)$ & \\
\hline$R V / L V=0.6-1.0$ & $41 / 150(27.33 \%)$ & $112 / 383(29.24 \%)$ & \\
\hline $\mathrm{RV} / \mathrm{LV}>1.0$ & $7 / 150(4.67 \%)$ & $18 / 383(4.70 \%)$ & \\
\hline Left Ventricular Wall Thickening & $22 / 150(14.67 \%)$ & $51 / 383(13.32 \%)$ & 0.683 \\
\hline Right Ventricular Wall Thickening & $16 / 150(10.67 \%)$ & $45 / 383(11.75 \%)$ & 0.724 \\
\hline IVC diameter & & & $0.008^{*}$ \\
\hline$<1.0$ & $33 / 150(22.00 \%)$ & 44/383 (11.49\%) & $0.002^{\star}$ \\
\hline $1.0-1.9$ & 74/150 (49.33\%) & $209 / 383(54.57 \%)$ & 0.276 \\
\hline$\geq 2.0$ & $43 / 150(28.67 \%)$ & 130/383 (33.94\%) & 0.242 \\
\hline IVC variability & & & $0.045^{\star}$ \\
\hline Large & $40 / 150(26.67 \%)$ & 71/383 (18.54\%) & $<0.001^{\star}$ \\
\hline Medium & $60 / 150(40.00 \%)$ & $146 / 383(38.12 \%)$ & 0.689 \\
\hline Small & $50 / 150(33.33 \%)$ & $166 / 383(43.34 \%)$ & $0.034^{\star}$ \\
\hline EF & & & 0.341 \\
\hline$>55 \%$ & $89 / 150(59.33 \%)$ & $242 / 383(63.19 \%)$ & \\
\hline $30-55 \%$ & $53 / 150(35.33 \%)$ & 130/383 (33.94\%) & \\
\hline
\end{tabular}




\begin{tabular}{|llll|}
$<30 \%$ & $8 / 150(5.33 \%)$ & $11 / 383(2.87 \%)$ & \\
\hline EF value & $53.536 \pm 14.8293$ & $56.171 \pm 14.3183$ & 0.168 \\
\hline Other Echocardiogram Abnomalities & $22 / 150(14.67 \%)$ & $46 / 383(12.01 \%)$ & 0.408 \\
\hline Echocardiography Score & $2.65 \pm 1.876$ & $2.59 \pm 1.939$ & 0.454 \\
\hline
\end{tabular}

Table 4 Clinical characteristics, outcomes and echocardiography manifestations stratified by survival status 28 days after ICU admission in patients with shock $(\mathrm{N}=\mathbf{2 8 8})$ 


\begin{tabular}{|c|c|c|c|}
\hline Variable & Non-survivors & Survivors & $\mathrm{P}$ \\
\hline Age $(y)$ & $59.69 \pm 18.011$ & $59.36 \pm 16.725$ & 0.891 \\
\hline HR & $108.84 \pm 25.129$ & $97.18 \pm 34.914$ & 0.015 \\
\hline MAP & $78.651 \pm 15.8965$ & $81.504 \pm 15.8202$ & 0.210 \\
\hline Lac & $5.960 \pm 5.4784$ & $3.091 \pm 2.7834$ & $<0.001^{\star}$ \\
\hline Oxygenation Index & $169.530 \pm 149.5003$ & $247.037 \pm 113.2802$ & 0.002 \\
\hline APACHEII & $24.63 \pm 8.884$ & $17.57 \pm 8.214$ & $<0.001^{\star}$ \\
\hline Lenth of MV (h) & $153.12 \pm 160.247$ & $150.66 \pm 432.526$ & 0.964 \\
\hline Lenth of ICU stay (d) & $26.23 \pm 125.323$ & $17.15 \pm 51.011$ & 0.389 \\
\hline Left Atrium Enlargement & $10 / 62(16.13 \%)$ & $30 / 226(13.27 \%)$ & 0.565 \\
\hline Right Atrium Enlargement & $8 / 62(12.90 \%)$ & $34 / 226(15.04 \%)$ & 0.672 \\
\hline Left Ventricle Enlargement & $8 / 62(12.90 \%)$ & $19 / 226(8.41 \%)$ & 0.282 \\
\hline Right Ventricle Enlargement & & & 0.280 \\
\hline $\mathrm{RV} / \mathrm{LV}<0.6$ & $39 / 62(62.90 \%)$ & $156 / 226(69.03 \%)$ & \\
\hline$R V / L V=0.6-1.0$ & $21 / 62(33.87 \%)$ & $56 / 226(24.78 \%)$ & \\
\hline $\mathrm{RV} / \mathrm{LV}>1.0$ & $2 / 62(3.23 \%)$ & $14 / 226(6.19 \%)$ & \\
\hline Left Ventricular Wall Thickening & $10 / 62(16.13 \%)$ & $29 / 226(12.83 \%)$ & 0.501 \\
\hline Right Ventricular Wall Thickening & $5 / 62(8.06 \%)$ & $15 / 226(6.64 \%)$ & 0.695 \\
\hline IVC diameter & & & 0.103 \\
\hline$<1.0$ & $15 / 62(24.19 \%)$ & $34 / 226(15.04 \%)$ & \\
\hline $1.0-1.9$ & $31 / 265(50.00 \%)$ & $106 / 226(46.90 \%)$ & \\
\hline$\geq 2.0$ & $16 / 62(25.81 \%)$ & $86 / 226(38.05 \%)$ & \\
\hline IVC variability & & & 0.276 \\
\hline Large & $14 / 62(22.58 \%)$ & $38 / 226(16.81 \%)$ & \\
\hline Medium & $26 / 62(41.94 \%)$ & $83 / 226(36.73 \%)$ & \\
\hline Small & $22 / 62(35.48 \%)$ & 105/226 (46.5\%) & \\
\hline EF & & & 0.203 \\
\hline$>55 \%$ & $29 / 62(46.77 \%)$ & $129 / 226(57.08 \%)$ & \\
\hline $30-55 \%$ & $25 / 62(40.32 \%)$ & $81 / 226$ (35.84\%) & \\
\hline
\end{tabular}




\begin{tabular}{|llll|}
\hline$<30 \%$ & $8 / 62(12.90 \%)$ & $16 / 226(7.08 \%)$ & \\
\hline EF value & $49.153 \pm 16.9019$ & $53.569 \pm 15.6674$ & 0.136 \\
\hline Other Echocardiogram Abnomalities & $14 / 62(22.58 \%)$ & $28 / 226(12.39 \%)$ & $0.044^{\star}$ \\
\hline Echocardiography Score & $3.03 \pm 1.916$ & $2.73 \pm 1.990$ & 0.685 \\
\hline
\end{tabular}

Table 5 Clinical characteristics, outcomes and echocardiography manifestations stratified by survival status 28 days after ICU admission in post-operation patients $(\mathrm{N}=759)$ 


\begin{tabular}{|c|c|c|c|}
\hline Variable & Non-survivors & Survivors & $\mathrm{P}$ \\
\hline Age $(y)$ & $64.50 \pm 12.298$ & $59.40 \pm 16.873$ & $0.010^{*}$ \\
\hline HR & $92.22 \pm 23.108$ & $87.44 \pm 20.966$ & $0.035^{\star}$ \\
\hline MAP & $89.203 \pm 16.3577$ & $92.787 \pm 16.0762$ & 0.144 \\
\hline Lac & $3.661 \pm 4.1440$ & $2.201 \pm 2.6389$ & $0.023^{*}$ \\
\hline Oxygenation Index & $266.145 \pm 181.8949$ & $304.781 \pm 102.4192$ & 0.372 \\
\hline APACHEII & $19.91 \pm 7.927$ & $11.59 \pm 6.732$ & $<0.001^{\star}$ \\
\hline Lenth of MV (h) & $118.00 \pm 128.558$ & $36.93 \pm 82.924$ & $<0.001^{\star}$ \\
\hline Lenth of ICU stay (d) & $9.74 \pm 7.350$ & $7.08 \pm 32.379$ & 0.578 \\
\hline Left Atrium Enlargement & $5 / 46(10.87 \%)$ & 72/713 (10.10\%) & 0.867 \\
\hline Right Atrium Enlargement & $9 / 46(19.57 \%)$ & $64 / 713(8.98 \%)$ & 0.263 \\
\hline Left Ventricle Enlargement & $3 / 46(6.52 \%)$ & $41 / 713(5.75 \%)$ & $<0.001^{\star}$ \\
\hline Right Ventricle Enlargement & & & 0.174 \\
\hline $\mathrm{RV} / \mathrm{LV}<0.6$ & $30 / 46(65.22 \%)$ & $550 / 713(77.14 \%)$ & \\
\hline$R V / L V=0.6-1.0$ & $15 / 46(32.61 \%)$ & $150 / 713(21.32 \%)$ & \\
\hline $\mathrm{RV} / \mathrm{LV}>1.0$ & $1 / 46(2.17 \%)$ & $13 / 713(1.82 \%)$ & \\
\hline Left Ventricular Wall Thickening & $12 / 46(26.09 \%)$ & 106/713 (14.87\%) & $0.042^{*}$ \\
\hline Right Ventricular Wall Thickening & $0 / 46(0 \%)$ & 19/713 (2.66\%) & $0.003^{*}$ \\
\hline IVC diameter & & & 0.491 \\
\hline$<1.0$ & $7 / 46$ (15.22\%) & $152 / 713(21.32 \%)$ & \\
\hline $1.0-1.9$ & $29 / 46(63.04 \%)$ & $442 / 713(61.99 \%)$ & \\
\hline$\geq 2.0$ & $10 / 46(21.74 \%)$ & $119 / 713(16.69 \%)$ & \\
\hline IVC variability & & & 0.716 \\
\hline Large & $13 / 46(28.26 \%)$ & $167 / 713(23.42 \%)$ & \\
\hline Medium & $20 / 46(43.48 \%)$ & $347 / 713(48.67 \%)$ & \\
\hline Small & $13 / 46(28.26 \%)$ & 199/713 (27.91\%) & \\
\hline EF & & & $<0.001^{\star}$ \\
\hline$>55 \%$ & $32 / 46$ (69.57\%) & $590 / 713(82.75 \%)$ & $0.024^{*}$ \\
\hline $30-55 \%$ & 10/46 (21.74\%) & 117/713 (16.41\%) & 0.348 \\
\hline
\end{tabular}




\begin{tabular}{|llll|}
$<30 \%$ & $4 / 46(8.70 \%)$ & $6 / 713(0.84 \%)$ & $<0.001^{\star}$ \\
\hline EF value & $53.379 \pm 16.0122$ & $61.467 \pm 11.0724$ & $0.014^{\star}$ \\
\hline Other Echocardiogram Abnomalities & $10 / 46(21.74 \%)$ & $66 / 713(9.26 \%)$ & $0.006^{\star}$ \\
\hline Echocardiography Score & $2.54 \pm 2.105$ & $1.84 \pm 1.681$ & $0.001^{\star}$ \\
\hline
\end{tabular}

Table 6 Risk factors of 28-day mortality

\begin{tabular}{|llll|}
\hline Item & R value & $\mathrm{P}$ value & $95 \% \mathrm{Cl}$ \\
\hline APACHEII & 0.903 & $<0.001$ & $0.873-0.933$ \\
\hline Length of MV & 0.994 & $<0.001$ & $0.991-0.997$ \\
\hline Length of ICU Stay & 1.159 & $<0.001$ & $1.083-1.242$ \\
\hline Oxygenation Index & 1.003 & 0.013 & $1.001-1.005$ \\
\hline Right ventricular wall thickening & 4.800 & 0.009 & $1.470-15.670$ \\
\hline IVC diameter & 1.756 & 0.024 & $1.075-2.868$ \\
\hline IVC variability & 1.797 & 0.017 & $1.112-2.904$ \\
\hline Echocardiography Score & 0.443 & 0.016 & $0.228-0.861$ \\
\hline
\end{tabular}

